\title{
Der hohe Blutdruck
}

Sudano, I ; Flammer, A ; Noll, G ; Lüscher, Thomas F

DOI: https://doi.org/10.1007/s00059-006-2759-0

Other titles: High blood pressure

Posted at the Zurich Open Repository and Archive, University of Zurich ZORA URL: https://doi.org/10.5167/uzh-36610

Journal Article

Published Version

Originally published at:

Sudano, I; Flammer, A; Noll, G; Lüscher, Thomas F (2006). Der hohe Blutdruck. Herz, 31(1):81-82.

DOI: https://doi.org/10.1007/s00059-006-2759-0 


\title{
Der hohe Blutdruck
}

\author{
Isabella Sudano, Andreas Flammer, Georg Noll, Thomas F. Lüscher ${ }^{1}$
}

Ein erhöhter Blutdruck (medizinisch: arterielle Hypertonie) ist ein wichtiger Risikofaktor für Erkrankungen der hirnversorgenden Gefäße, der Gefäße des Herz-Kreislauf-Systems sowie für die Entstehung eines Nierenleidens.

Internationale medizinische Fachgesellschaften haben Richtlinien festgelegt, nach denen der Blutdruck entsprechend seiner Höhe in einzelne Klassen eingeteilt wird (Tabelle 1).

Die Wahrscheinlichkeit für das Auftreten von Herz- und Kreislauf-Erkrankungen steigt mit der Höhe des systolischen und diastolischen Blutdrucks kontinuierlich an.

Problematisch ist, dass sich ein erhöhter Blutdruck schleichend entwickelt, ohne dass die Patienten etwas davon spüren, und dies macht ihn so gefährlich. Die Mehrheit der Betroffenen bemerkt jahrelang keinerlei Beschwerden, obwohl der Bluthochdruck die Gefäße und Organe schädigt und das Risiko für Folgeerkrankungen massiv erhöht.

\section{Wie kann ich meinen Blutdruck messen?}

Die Blutdruckmessung spielt für die Diagnose und Behandlung des Bluthochdrucks eine wesentliche Rolle.

\section{Welche Geräte sollten benutzt werden, um den Blutdruck zu messen?}

In der Arztpraxis wird der Blutdruck am häufigsten mit einer aufblasbaren Oberarmmanschette, welche mit einem Quecksilbersphygmomanometer oder mit einem Manometer verbunden ist, gemessen. Dabei wird die Manschette so lange aufgepumpt, bis die Arterie kein Blut mehr durchlässt. Durch das Ablassen der Luft vermindert sich der Druck in der Manschette wieder, und je nach Blutdruck strömt jetzt Blut durch das Gefäß, welches mittels eines Stethoskops abgehört werden kann. Das erste hörbare Geräusch entspricht dem oberen, systolischen Blutdruck, das Verschwinden des Geräuschs dem unteren, diastolischen Blutdruck.

Am zuverlässigsten sind die Blutdruckmesssysteme mit Quecksilber, welche jedoch aufgrund ökologischer Überlegungen immer weniger gebraucht werden. Sie gelten wegen ihrer Ge-

\footnotetext{
${ }^{1}$ Kardiovaskuläres Zentrum, Kardiologie,

UniversitätsSpital Zürich, Schweiz.
}

Herz 2006;31:81-2

DOI $10.1007 /$ s00059-006-2759-0 nauigkeit immer noch als Goldstandard bzw. als Referenzsystem. Manometer mit Luft sind eine gute Alternative, verlieren aber bei längerem Gebrauch mit der Zeit an Messgenauigkeit.

Mittlerweile stellen auch vollautomatische Blutdruckmessgeräte eine gute Alternative für eine Blutdruckmessung dar. Diese sind zwar weniger genau, haben aber den Vorteil, dass

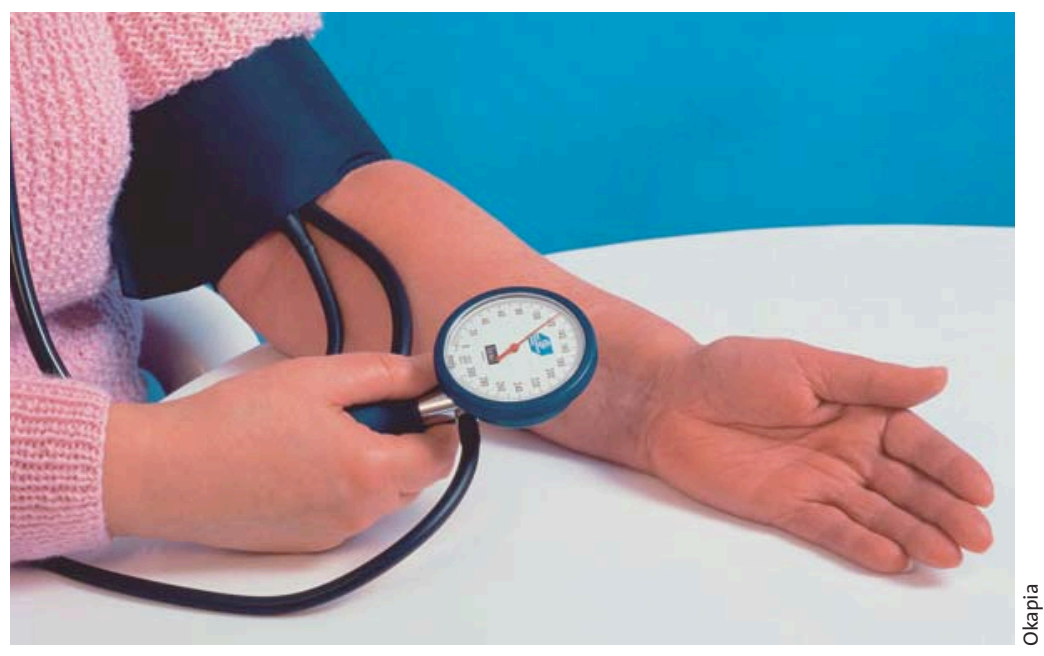

die Patienten ihren Blutdruck selbständig messen können. Nützlich ist auch, dass diese Blutdruckmessungen zu verschiedenen Tageszeiten und nicht in der Praxisumgebung durchgeführt werden können, so dass die Werte eher den Blutdruck im Alltag widerspiegeln. Wichtig ist jedoch, den Patienten den Messvorgang genau zu erklären (am besten im Rahmen einer Schulung) und beim Kauf darauf zu achten, dass die Geräte klinisch geprüft sind.

Tabelle 1. Aktuell gültige Einteilung des Bluthochdruckes gemäß den Richtlinien internationaler Fachgesellschaften.

\begin{tabular}{lll}
\hline Klassifikation & $\begin{array}{l}\text { Systolischer Blutdruck } \\
(\mathbf{m m H g})\end{array}$ & $\begin{array}{l}\text { Diastolischer Blutdruck } \\
(\mathbf{m m H g})\end{array}$ \\
\hline Optimal & $<120$ & $<80$ \\
Normal & $120-129$ & $80-84$ \\
${ }_{\text {"Hoch" }}$-normal & $130-139$ & $85-89$ \\
Bluthochdruck Grad I (leicht) & $140-159$ & $90-99$ \\
Bluthochdruck Grad II (mittelschwer) & $160-179$ & $100-109$ \\
Bluthochdruck Grad III (schwer) & $\geq 180$ & $\geq 110$ \\
Isolierter systolischer & $\geq 140$ & $<90$ \\
Bluthochdruck & & \\
\hline
\end{tabular}


Tabelle 2. Messrichtlinien.

- Der Blutdruck sollte in sitzender Position unter Beachtung der richtigen Armposition ermittelt werden. Der Messpunkt sollte grundsätzlich auf Herzhöhe sein, was anatomisch etwa der Brusthöhe entspricht

- Die Messung sollte nach 5-minütiger Ruhe in einem stillen Raum erfolgen. Vorausgegangene seelische oder körperliche Belastungen, Umgebungstemperatur sowie Alkohol- oder Nikotinkonsum verändern die Werte

- Es sollte eine Standardmanschette (35 cm Breite) bei Oberarmumfang bis zu $32 \mathrm{~cm}$ verwendet werden. Bei über $32 \mathrm{~cm}$ Oberarmumfang sollte eine breitere und für Kinder eine schmalere Manschette gebraucht werden

- Der systolische Blutdruck entspricht dem ersten hörbaren Geräusch, der diastolische Blutdruck entspricht dem Verschwinden des Geräuschs. Der Blutdruck sollte auf $2 \mathrm{mmHg}$ genau bestimmt werden, ein Auf- oder Abrunden auf $5 \mathrm{mmHg}$ ist zu vermeiden

- Beim ersten Arztbesuch sollte der Blutdruck an beiden Armen gemessen werden, und bei Differenzen $>20 / 10 \mathrm{mmHg}$ wird eine Abklärung durch einen Gefäßspezialisten erfolgen

- Zwischen aufeinander folgenden Messungen sollten wenigstens 3 min verstreichen

- Zur Beurteilung der Blutdruckhöhe sollte der Mittelwert aus zwei bis drei aufeinander folgenden Messungen genommen werden

Korrespondenzanschrift

Dr. Isabella Sudano Kardiologie

UniversitätsSpital

Zürich

Rämistraße 100

8091 Zürich

Schweiz

Telefon (+41/44)

250-4095,

Fax -4090

E-Mail: isabella.

sudano@usz.ch
Wichtig bei allen Messmethoden ist, dass die Richtlinien eingehalten werden (Tabelle 2).

Ein Blutdruck von 135/85 $\mathrm{mmHg}$ für den selbstgemessenen Blutdruck kann als normal angesehen werden

\section{4-h-Blutdruckmessung}

Eine weitere wichtige Anwendung vollautomatischer Blutdruckmesssysteme ist die Messung über $24 \mathrm{~h}$, um den Blutdruck auch nachts und während der Arbeit überwachen zu können. Durch die vielen Messungen (alle $15 \mathrm{~min}$ am Tag und alle 30 min in der Nacht) können wertvolle Informationen über das Blutdruckverhalten in speziellen Situationen erhalten werden. So erlaubt diese Messung z.B. die Aufdeckung einer sog. Weißkittelhochdrucks, d.h. eines Bluthochdrucks, der nur in der Praxis, nicht jedoch im alltäglichen Leben besteht. Auch ein gestörter Tag-Nacht-Rhythmus kann dokumentiert werden. Außerdem erlaubt eine Messung über $24 \mathrm{~h}$ eine bessere Einschätzung des individuellen Herz-Kreislauf-Risikos und eine bessere Überwachung der Behandlung.

\section{Mein Blutdruck ist erhöht.}

\section{Was sollte weiter abgeklärt werden?}

Wenn ein Bluthochdruck nach mehreren Blutdruckmessungen gesichert ist, besteht das Ziel der weiteren Diagnostik darin, Folgekrankheiten und Risikofaktoren bzw. Begleiterkrankungen zu erfassen. Wichtig ist auch die Erfassung eines Bluthochdrucks, der durch eine meist behandelbare Erkrankung ausgelöst wurde.

Grundlage der Diagnostik sind die Krankengeschichte und die körperliche Untersuchung, die Bestimmung der Blutsalze (Natrium und Ka- lium), Nierenfunktion sowie Blutfette und -zucker. Zusätzlich sollten eine Urinuntersuchung, ein EKG und ggf. ein Bauch-Ultraschall durchgeführt werden. Je nach Fall wird der Spezialist weitere Untersuchungen anordnen.

\section{Wie sollte mein Blutdruck behandelt} werden?

Grundsätzlich kann der Bluthochdruck mittels Allgemeinmaßnahmen oder mit Medikamenten behandelt werden.

\section{Behandlung ohne Medikamente}

- Gewichtsreduktion.

- Regelmäßige körperliche Betätigung.

- Kochsalzarme Diät (bei gewissen Patienten).

- Einschränkung des Alkoholkonsums (maximal 2-3 Gläser Wein, 2 kleine Gläser Bier pro Tag).

- Individuelle Verfahren zur Stressbewältigung.

- Ausgewogene Ernährung (mediterrane Kost).

- Senkung des gesamten Herz-KreislaufRisikos (z.B. kein Nikotinkonsum, fettarme Diät).

\section{Behandlung mit Medikamenten}

Die Wahl des richtigen Medikaments sollte nicht allein aufgrund seiner blutdrucksenkenden Wirkung und der Verträglichkeit erfolgen. Die Behandlung verfolgt das Ziel, mit möglichst wenigen Nebenwirkungen das erhöhte Herz-Kreislauf-Risiko wirksam zu senken. Je nach Begleiterkrankungen, bereits vorhandenen Schäden wegen des hohen Blutdrucks (z.B. Herzmuskelvergrößerung) oder Verträglichheit der einzelnen Substanzen muss für jeden Patienten eine individuell angepasste Behandlung zusammengestellt werden.

Wie niedrig sollte der Blutdruck sein? Grundsätzlich ist eine Blutdrucknormalisierung anzustreben. Unter Ruhebedingungen sollte der Blutdruck systolisch $<140 \mathrm{mmHg}$ und diastolisch $<90 \mathrm{mmHg}$ liegen. Tiefere Blutdruckwerte gelten für Kinder und Jugendliche sowie während der Schwangerschaft. Bei Diabetikern ist die konsequente Senkung des Blutdrucks auf Werte $<130 / 80 \mathrm{mmHg}$ zu empfehlen, insbesondere wenn in der Urinuntersuchung eine erhöhte Eiweißausscheidung festgestellt wurde oder bereits eine Nierenerkrankung besteht. In vielen Studien konnte nachgewiesen wird, dass mit einer konsequenten Behandlung des hohen Blutdrucks das Risiko für Herzinfarkte, Schlaganfälle und für die Entwicklung eines Herz- oder Nierenversagens deutlich vermindert werden kann. 